\title{
Enhancing the Life Time of a Wireless Sensor Network by Ranking and Recovering the Fault Nodes
}

\author{
Reghunath E V ${ }^{1}$, Pankaj Kumar ${ }^{2}$, Ashok Babu ${ }^{3}$ \\ ${ }^{1}$ M.Tech Student, ${ }^{2}$ Asst. Professor \\ ${ }^{1,3}$ School of Computer Sciences, M G University, Kerala, India \\ ${ }^{2}$ Asst. Professor Computer Science Dept. FISAT, Kerala, India
}

\begin{abstract}
Wireless sensor networks consist of set of specially designed electronic devices called nodes. Each sensor node has an ability of collecting information from the environment it is used for. It will collect environmental conditions such as temperature, sound, pressure, vibration, motion from different locations. The performance of the wireless sensor network may vary due to certain situations like the breaking of the data routing path, data loss during transmission, reducing the battery power of nodes during data transmission. The sensor node will consume more battery power at the time of data transmission as well as reception. Hence the battery power will decrease to a limit, means the node becomes fault or unusable. It should be replaced. Here we are proposing a new method for finding the least number of fault nodes for replacement.
\end{abstract}

Keywords - Grade Diffusion Algorithm, Direct Diffusion Algorithm, Genetic Algorithm, Fault Node Recovery Algorithm

\section{INTRODUCTION}

In wireless sensor network (WSN), the sensor nodes are deployed over a geographical area for collecting informations. Usually these informations are sending to a base station called Sink node [3] [4]. The wireless sensor network sometimes affected by unexpected problems due to various situations like hardware issues, environmental problems, software issues. The sensor nodes are powered by tiny batteries. So the nodes may fail due to the discharging of battery power during the data transmission as well as data reception. The failure of the battery is a major problem in WSN. Failure in the hardware/software inside the node also affects proper working of wireless sensor nodes. Fig 1 shows a wireless sensor network with fault node due to battery loss.

\section{PROBLEM DESCRIPTION}

The nodes in the wireless sensor networks become faulty due to several factors like hardware/software failure, network error, congestion in the network, unexpected failure in routes. The life time of the node is depends upon the battery power. The sensor node will use most of its battery power for data transmission as well as reception [1]. So, whenever data transfer rate is increased, the battery power will be gradually decreased, and after a while the node becomes unusable due to low battery.

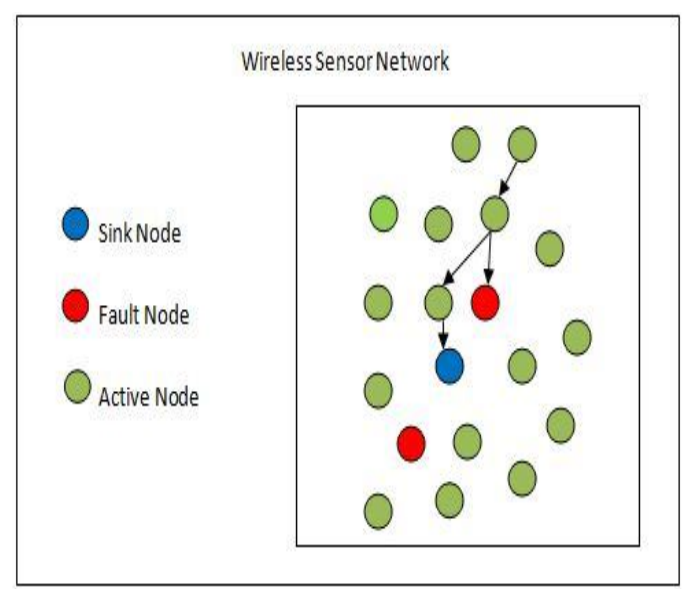

$$
\text { Fig. } 1 \text { Fault node occurrence }
$$

III. RELATED WORKS

Energy efficient routing in WSN is an important thing. The conventional approach for the routing includes the Directed Diffusion (DD) [5] algorithm and the Grade Diffusion (GD) [6] algorithm. A fault node recovery algorithm [1] is proposed by Hong-Chi Shih et al, with the goal of replacing fewer sensor nodes, when fault node occurred in the network.

\section{A. Directed Diffusion Algorithm}

The directed diffusion algorithm (DD) was proposed by C.Intanagonwiwat et al. is a query driven transmission protocol. The informations which are collected by the nodes are transmitted only when it matches the query message in the form of attribute-value pairs to other sensor nodes in the network by broadcasting it. The sensor nodes will sends the collected data back to the sink node only when the query matches.

\section{B. $\quad$ Grade Diffusion Algorithm}

The Grade Diffusion (GD) algorithm is presented by H.C Shih et al. which improve the ladder diffusion algorithm using ant colony optimization [2] that clearly used for wireless sensor routing. The GD algorithm is not only for routing but 
also used for identify the neighbor nodes of all sensor nodes. It also records some information about the data relay and grade values for all nodes. Hence the GD algorithm updates the routing path in real time.

\section{Fault Node Recovery Algorithm}

Fault Node Recovery (FNR) Algorithm is based on the Grade Diffusion algorithm combined with the Genetic Algorithm [1]. The FNR algorithm also creates grade value, routing table, neighbor nodes for each sensor nodes in the network. The Grade Diffusion algorithm will create the route between the nodes and the sink node. Whenever fault node occurred in the network, the genetic algorithm executes and finds lest number of sensor nodes which is to be replaced.

The sensor nodes which are nearer to the sink node have more importance in the network. They are the inner nodes in the network. The outer nodes send data to the sink node through inner nodes. Hence the priority in fault node replacement should be given to the inner nodes. In order to do this, a ranking system is implemented in the proposed method. Each inner node has ranks as it appears closer to the sink node. The replacement of nodes is also done by verifying the rank of the node. The higher ranked node should get a priority in replacement, because other nodes will use it as a path to sink node

\section{CURRENT APPROACH}

In the current approach, a ranking system is implemented for providing sufficient ranks to the sensor nodes according to its available incoming and outgoing routes. The sensor nodes send data to the sink node through the routes assigned by the Grade Diffusion algorithm. Non - functioning nodes are replaced by functional nodes by using the $\mathrm{K}_{\mathrm{th}}$ in the Eq (1)

$$
\begin{aligned}
& K_{t h}=\sum_{g=1}^{\max (\text { grade })} P_{i} \\
& P_{i}=\left\{\begin{array}{l}
1, \frac{N_{i}^{\text {now }}}{N_{i}^{\text {original }}}<\beta \\
0, \text { otherwise }
\end{array}\right.
\end{aligned}
$$

In (1) $\mathrm{N}_{i}^{\text {original }}$ is the number of sensor nodes with grade value $\mathrm{i} . \mathrm{N}_{i}^{\text {now }}$ is the number of sensor nodes still working with grade value i. $\beta$ is the value set by the user and it will be between 0 and 1. The Grade Diffusion method uses the Ladder Diffusion Algorithm using Ant Colony Optimization (LDACO) [2] for routing. The sink node broadcasts a ladder creating packet with grade value of one. This means the sensor nodes which receives the ladder creating packets, transmits data to the sink node with a hope of one. Then these nodes will increments the grade value and broadcasts again. After a while all nodes have its grade value. In order to implement this procedure, neighbor nodes of all sensor nodes must be identified. Hence each node has its own neighbor node list and from this list the node will select the neighbor nodes for broadcasting packets. Whenever the events occur the sensor nodes will sends the data back to the sink node. The routes to the sink node are created during the ladder creating phase. A node creates path through neighbor nodes. Hence it has different paths to same destination. From these, one of the optimum routes should be selected. For this the fitness function is calculated by the Eq (2).

$$
P_{(i j)}=r\left(\alpha_{1}+\alpha_{2} d_{(i j)}^{n}\right)+N_{j}
$$

In Eq (2), $P_{(i j)}$ is the energy consumption after node $i$ transmit data to node $\mathrm{j} . \mathrm{r}$ is the data transfer rate of the sensor nodes. $\mathrm{d}_{(\mathrm{i} j)}$ is the Euclidian distance between node $\mathrm{i}$ and node j. $\alpha_{1}$ is the distance-independent parameter, $\alpha_{2}$ is the distancedependent parameter. The selecting rate for all backup node found using $\mathrm{Eq}(3)$.

$$
U_{k}(i, j)=\frac{[\tau(i, j)] \cdot\left[P_{(i, j)}^{-1}\right]^{\beta}}{\sum_{J_{(i)}}[\tau i, j] \cdot\left[P_{(i, j)}^{-1}\right]^{\beta}} \quad, j \in j(i)
$$

In Eq (2), $U_{k}(i \mathrm{j})$ is the probability that data on node $i$ choses to move to node $\mathrm{j}$. $\tau$ ( $\mathrm{i} \mathrm{j}$ ) is the pheromone between node $\mathrm{i}$ and node $\mathrm{j} . \mathrm{P}_{(\mathrm{i})}$ is the energy consumption of node $\mathrm{i}$ and node $\mathrm{j}$ from $\mathrm{Eq}(2)$. The $\beta$ is an evaluation parameter of pheromone on paths. The ant colony technology provides pheromone for each selected paths. The pheromone will evaporate from the path after a particular time. Hence the pheromone update can be done by using Eq (4). The pheromone update is required for keeping the path alive.

$$
\tau_{(x y)} \leftarrow(1-p) \tau_{(x y)}+\sum_{k} \Delta \tau_{x y}^{k}
$$

In Eq (4) $\tau_{(x, y)}$ is the amount of pheromone deposited between node $\mathrm{x}$ and node $\mathrm{y} . \rho$ is the pheromone evaporation coefficient. $\Delta \tau_{(\mathrm{x} y)}{ }^{\mathrm{k}}$ is the amount of pheromone deposited by $\mathrm{K}_{\mathrm{th}}$ data transmission. In the proposed method, the optimum solution to the replacement of the fault node is found by the genetic algorithm. Hence the least number of nodes can be replaced by using this algorithm. A ranking system is implemented in this approach. So each inner node has ranks as it appears closer to the sink node. The replacement of nodes is also done by verifying the rank of the node. The higher ranked node should get a priority in replacement, because other nodes will use it as a path to sink node. After execution of the genetic algorithm there will be a set of fault nodes for replacement. The replacement cost can be reduced with the help of the ranking system. The ranked nodes will get a priority than the nodes far from the sink node. Hence the network life time will increase.

In order to perform this, the $\mathrm{K}_{\mathrm{th}}$ is calculated according to $\mathrm{Eq}$ (1) for each data transfer to the sink node. If it is larger than zero, the nonfunctioning nodes selected by the genetic algorithm will replace it with functioning nodes. The genetic algorithm follows 5 steps. They are initialization, evaluation, selection, crossover and mutation [7].

\section{a) Initialization}

Initialization is the first step of the genetic algorithm. Chromosomes are generated in this step. A chromosome is a collection of genes and it is arranged in a string like structure. 
The user will determine the initial population size, so the number of chromosome is depends upon it. The length of the chromosome shows the number of non-functioning sensor nodes. The gene elements are either 0 or 1 . A 1 means the faulty node should be replaced and a 0 means the node will not be replaced. Figure 3 represents a chromosome structure, in which the node numbers are of non-functioning nodes.

\begin{tabular}{|l|r|r|r|r|r|r|r|r|r|}
\hline 9 & 15 & 23 & 32 & 37 & 20 & 82 & 67 & 58 & 21 \\
\hline $\mathbf{1}$ & $\mathbf{0}$ & $\mathbf{0}$ & $\mathbf{0}$ & $\mathbf{1}$ & $\mathbf{1}$ & $\mathbf{0}$ & $\mathbf{1}$ & $\mathbf{0}$ & $\mathbf{1}$ \\
\hline
\end{tabular}

Fig. 3 Chromosome and Its Gene

Here, 10 nonfunctioning nodes $8,6,12,20,16,28,35$, $33,40,3$ are selected and arranged for a chromosome structure. Gene value is given for each node.

\section{b) Evaluation}

In GA, the fitness of a chromosome is evaluated using a fitness function. Most routing paths should be reused and replace fewest sensor nodes. So the fitness function should be calculated by using Eq (5).

Here,

$$
f_{n}=\sum_{g=1}^{\max (\text { grade })} \frac{M_{J} \times T_{p}^{-1}}{N_{J} \times T_{N}^{-1}} \times j^{-1}
$$

$\mathrm{M}_{\mathrm{J}}=$ the number of reusable routing paths from a sensor Node with grade $\mathrm{i}$

$\mathrm{N}_{\mathrm{J}}=$ the number of replaced sensor nodes with grade $\mathrm{i}$.

$\mathrm{T}_{\mathrm{P}}=$ total number of routing paths in network.

$\mathrm{T}_{\mathrm{N}}=$ total number of sensor nodes in the network.

\section{c) Selection}

The selection process is done by using the result of the fitness function. This step will help us to find out the low fitness valued chromosome and eliminate them. Selection step is shown in Fig. 4. Collection of chromosomes for the selection procedure is generated during the setting up of initial population. The initial population is set up by the user. According to the initial population set of combinations will be prepared for creating the chromosomes. Thus the chromosome gene value is the combination selected from the initial population

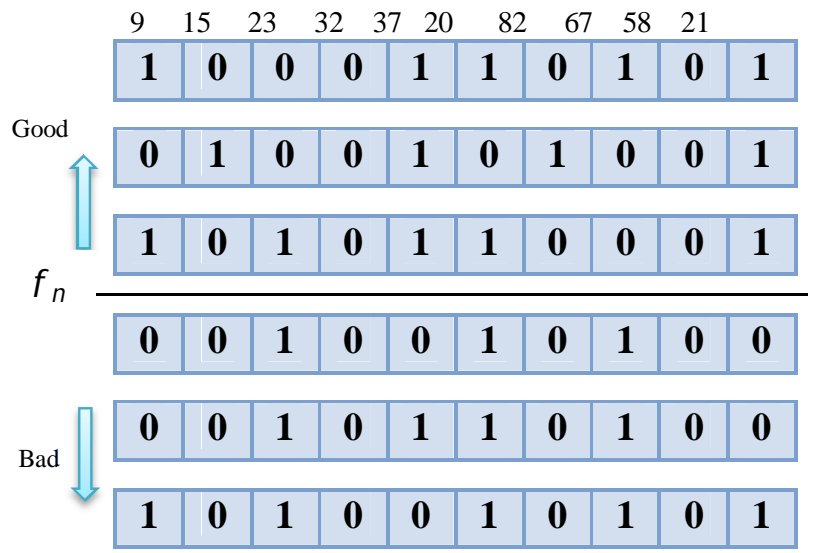

Fig. 4 Selection Procedure
There will be a limit for the fitness value and chromosomes below that limit are not selected. The rest are good and will be moved to chromosome mating pool. The selection step is essential for the next step (crossover).

\section{d) Crossover}

The crossover operation is done by setting a crossover point over the chromosome. For this two individual chromosomes are taken from the mating pool. Here one point crossover method is used. The crossover limit is setup as shown in Fig. 5.

\begin{tabular}{|l|l|l|l|l|l|l|l|l|l|}
\hline \multicolumn{1}{|c|}{15} & 23 & 32 & 37 & 20 & 82 & 67 & 58 & 21 \\
\hline $\mathbf{1}$ & $\mathbf{0}$ & $\mathbf{0}$ & $\mathbf{0}$ & $\mathbf{1}$ & $\mathbf{1}$ & $\mathbf{0}$ & $\mathbf{1}$ & $\mathbf{0}$ & $\mathbf{1}$ \\
\hline
\end{tabular}
\begin{tabular}{|l|l|l|l|l|l|l|l|l|l|}
\hline 0 & 1 & 0 & 0 & 1 & 0 & 1 & 0 & 0 & 1 \\
\hline
\end{tabular}

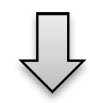

\begin{tabular}{|l|l|l|l|l|l|l|l|l|l|}
9 & 15 & 23 & 32 & 37 & 20 & 82 & 67 & 58 & 21 \\
\hline 1 & 0 & 0 & 0 & 1 & 0 & 1 & 0 & 0 & 1 \\
\hline 0 & 1 & 0 & 0 & 1 & 1 & 0 & 1 & 0 & 1 \\
\hline
\end{tabular}
\begin{tabular}{|l|l|l|l|l|l|l|l|}
\hline 0 \\
\hline
\end{tabular}

Fig. 5 Crossover Step

As shown in the figure the genes after the crossover limit is selected and they will flip over the other and thereby creating a pair of child chromosomes

\section{e) Mutation}

The mutation adds variation in the next generation of the chromosome. In mutation a node selected randomly and the gene value of the selected node is inverts. Fig 6 shows the mutation step.

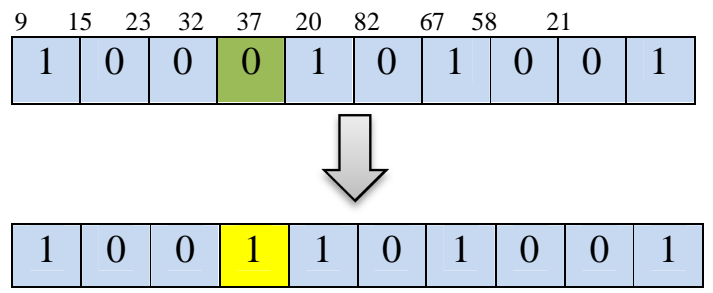

Fig 6 Mutation Step

After several iterations a chromosome with the best fitness value will obtain. From this the selected nodes can be replaced by verifying the rank of the corresponding nodes. The priority is given to high ranked nodes for the replacement. But alternative path should be selected to transfer the data to the sink node. For this the available good neighbor nodes are selected and a new path will form. 


\section{SIMULATION}

The algorithms are executed with the help of a simulator that presents a visual environment for the deployment of wireless sensor nodes. The sensor nodes are deployed over the simulator on corresponding co-ordinates. The co-ordinates can be considered as the geographical location of the nodes in the original environment. The energy levels of each node are showed. Initially all nodes have full energy. The sink node is placed at the center of the canvas Fig 7 shows the python canvas that includes the wireless sensor node setup.

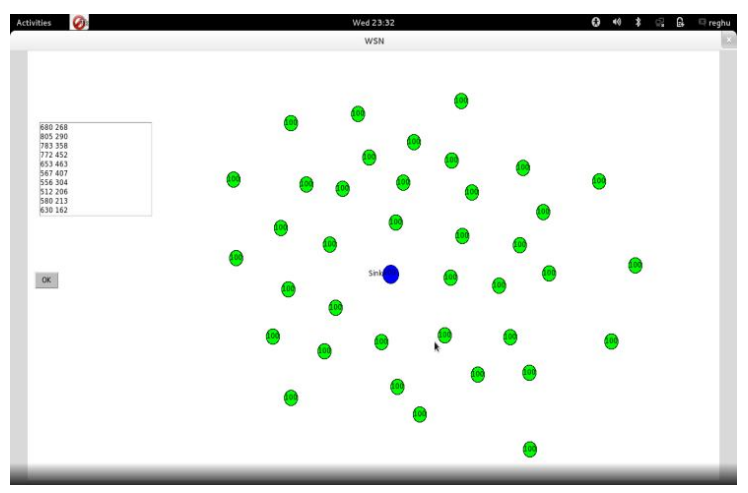

Fig. 7 Sensor nodes are on geographical positions

Neighbor nodes of all nodes are found for proper routing and the data transfer is done by selecting the proper neighbor nodes. After several data transfer some nodes will become fault nodes. At the time of data transfer the energy levels of the participating nodes will be decreased according to the data transfer rate. Fig 8 shows the data transfer procedure. The red colored nodes are fault nodes. The ratio in between currently functioning nodes and all sensor nodes are calculated and according to this the genetic algorithm will be executed. Genetic algorithm will figure out fault nodes. This paper presents a ranking system for all wireless sensor nodes. Hence after the execution of the genetic algorithm the ranked nodes are treated first.

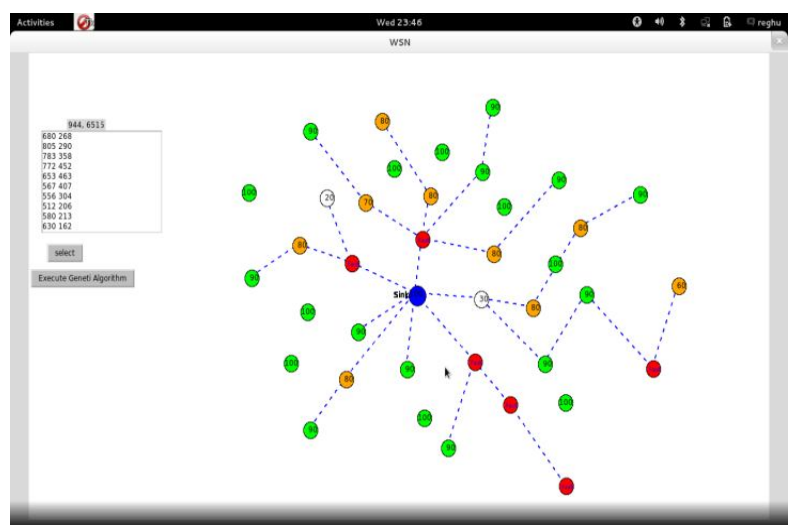

Fig. 8 Data transmission \& Fault node occurrences
There will be a collection of fault nodes after the algorithm execution. According to the fault nodes, the route will be replaced using readily available good nodes. The ranked nodes will be shown separately by its position. Low ranked nodes are far from the sink node. The high ranked nodes are commonly used nodes. The rank is measured for all nodes from grade 1 to the maximum grade.

From a set of 40 wireless sensor nodes, the genetic algorithm will choose 5 fault nodes each time and from this 3 high ranked nodes nearer to sink will get a priority in replacement.

\section{CONCLUSION}

This paper proposes a ranking system for all wireless nodes according to the incoming and outgoing data paths. The nodes nearer to the sink node will have high ranks. So the high ranked nodes will get the priority in replacement. Thus the cost of replacing sensor nodes far from the sink node can be reduced by selecting the ranked node at the time of replacing. This will save the lifetime of the wireless sensor network. Also alternate paths will be selected when a node becomes a fault node. The path selected will be the energy efficient one. Hence the data will receive its correct destination in low cost.

\section{ACKNOWLEDGMENT}

This work was conducted in Centre for High Performance Computing Lab FISAT, Angamaly, Kerala and all facilities provided by M G University, Kerala. We express our special thanks to Dr. R. Vijayakumar, Professor, School of Computer Sciences, M.G University.

\section{REFERENCES}

[1] Hong-Chi Shih, Jiun-Huei Ho, Bin-Yih Liao and Jeng -Shyang Pan, "Fault Node Recovery Algorithm for a wireless sensor network." IEEE Sensors Journal, Vol-13, No. 7, July2013

[2] J. H. Ho, H. C. Shih, B. Y. Liao and S. C. Chu," A Ladder Diffusion Algorithm Using Ant Colony Optimization for Wireless Sensor Networks", Information Sciences, (2011)

[3] Z. He, B. S. Lee, and X. S. Wang, "Aggregation in sensor networks With a user-provided quality of service goal," Inf. Sci., vol. 178, no.9, PP. $2128-2149,2008$.

[4] T. P. Hong and C. H. Wu, "An improved weighted clustering algorithm For determination of application nodes in heterogeneous sensor Networks" J. Inf. Hiding Multimedia Signal Process, vol. 2, no. 2, pp. 173-184, 2011

[5] C Intanagonwiwat, R. Govindan, D. Estrin, J. Heidemann and F. Silva, "Directed diffusion for wireless sensor networking," IEEE/ ACM Trans. Netw., vol. 11, no. 1, pp. 2-16, Feb. 2003

[6] E. M. Royer and C. K. Toh, "A review of current routing protocols for Ad -hoc mobile networks, ” IEEE Personal Commun.., vol. 6, no. 2, pp. 46-55, Apr. 1999.

[7] M.Gen and R. Cheng, Genetic Algorithms and Engineering Design. New York, NY, USA: Wiley, 1997. 